Wiederherstellung eines normalen psychosozialen Funktionsniveaus und die Teilhabe am Sozialleben für die Patienten eine höhere Bedeutung habe als eine rein symptomatische Remission, müssen diese Aspekte bei der Bewertung des Therapieerfolges berücksichtigt werden. Ein geeignetes Instrument zur Erfassung der Funktionsbeeinträchtigungen sei die Sheehan Disability Scale (SDS), so Demyttenaere.

Professor Göran Hajak, Klinikum Bamberg, wies darauf hin, dass Agomelatin (Valdoxan ${ }^{\circledR}$ ) eine ausgeprägte Wirksamkeit auf alle relevanten Kernsymptome der Depression habe, einschließlich Stimmung, Angst, Schlaf, Arbeit und Aktivitäten. Zwei neue Metaanalysen [Kasper S et al. Int Clin Psychopharmacol
2013; 28: 12-192; Demyttenaere K et al. CNS Spectrum 2013; 11: 1-8] zeigten eine überzeugende Datenlage für Agomelatin bezüglich Symptomveränderung und Responserate im Vergleich zu anderen Antidepressiva. Außerdem führe Agomelatin zu einer schnell einsetzenden Verbesserung des psychosozialen Funktionsniveaus und der Anhedonie, so Hajak. Auch über 65-jährige Patienten sowie depressive Patienten mit ausgeprägten Angstsymptomen können von der Substanz profitieren.

Abdol A. Ameri, freier Medizinjournalist

Satellitensymposium „Recovering usual functioning in depressed patients with agomelatine", 21st European Congress of Psychiatry (EPA),

Nizza, 8.4.2013; Veranstalter: Servier

\title{
Alkoholabhängigkeit
}

\section{Zielvorgabe: Reduktion des Alkoholkonsums}

Abstinenz-orientierte Behandlungsstrategien waren bisher der "Goldstandard“ in der Therapie der Alkoholabhängigkeit. Aber es gelinge nur selten, dieses Ziel zu erreichen, unterstrich Professor Karl Mann, Zentralinstitut für Seelische Gesundheit Mannheim. Eine weitaus realistischere und patientenfreundlichere Therapieoption sei die Reduktion des Gesamtalkoholkonsums.

Medikamente wie der Opioidrezeptorantagonist Nalmefen (Selincro ${ }^{\varpi}$ ) können Patienten dabei unterstützen, ihren Alkoholkonsum unter die Grenze von $60 \mathrm{~g} / \mathrm{d}$ (Männer) beziehungswesie $40 \mathrm{~g} / \mathrm{d}$ (Frauen) (hohes Risikoniveau nach WHO) zu senken.

Die Konsumreduktion durch Nalmefen (18 mg) als Bedarfsmedikation wurde in drei randomisierten, placebokontrollierten Doppelblindstudien bei insgesamt etwa 2.000 alkoholabhängigen Patienten untersucht [Mann K et al. Biol Psychiatry. 2013; 73: 706-13; Gual A et al. und Van den Brink W et al., Poster presented at the RSA Scientific Meetings, San Francisco, 2012].

Sowohl der monatliche Gesamtalkoholkonsum als auch die Anzahl der Tage mit starkem Konsum (Heavy Drinking Days, HDD, definiert als $\geq 60 \mathrm{~g} / \mathrm{d}$ bei Männern bzw. $\geq 40 \mathrm{~g} / \mathrm{d}$ bei Frauen) wur- den in der Verumgruppe signifikant $(\mathrm{p}<0,05)$ stärker reduziert als in der Kontrollgruppe. „Die Patienten konnten ihren Alkoholkonsum reduzieren und die Reduktion auch langfristig aufrechterhalten“, betonte Mann. Der therapeutische Nutzen von Nalmefen spiegelte sich zudem in einer deutlichen Verbesserung des klinischen Gesamtzustands wi$\operatorname{der}(\mathrm{p}<0,05$ vs. Kontrollgruppe).

Dass eine Reduktion des Alkoholkonsums tatsächlich klinisch relevant ist, belegte Chaim Jellinek, niedergelassener Allgemeinmediziner aus Berlin, an folgendem Beispiel: Ein Patient, der jeden Abend eine Flasche Wein trinkt habe ein Sterblichkeitsrisiko infolge von alkoholassoziierten Verletzungen von $18 \%$. Wenn er seinen Alkoholkonsum auf $40 \mathrm{~g}$ Reinalkohol pro Tag reduziert, sinke das Risiko auf $2 \%$. Jellinik sieht in der neuen Therapieoption auch eine Chance, alkoholabhängige Patienten schon wesentlich früher in ein Behandlungssetting einzubinden.

Abdol A. Ameri, freier Medizinjournalist

Pressekonferenz „Blick hinter die Behandlungstür: Therapie der Alkoholabhängigkeit als Herausforderung für Patient und Arzt", Berlin, 22.4.2013

Veranstalter: Lundbeck GmbH

\section{Unabhängige MS-Studie}

Nach der vollständig vom National Institute of Health (NIH) finanzierten doppelblinden, randomisierten CombiRx-Studie [Lublin F et al. Annals of Neurology 2013. DOI: 10.1002/ana. 23863] hat bei der Behandlung der Multiplen Sklerose die Kombination von Glatirameracetat (Copaxone ${ }^{\circledast}$ ) und Interferon beta-1a im Head-to-headVergleich zur Monotherapie mit Glatirameracetat keinerlei klinische Vorteile, ist aber der Monotherapie mit Interferon überlegen.

Nach Informationen von Teva

\section{Portfolio erweitert}

Das Produktportfolio von neuraxpharm ist um Entacapon-neuraxpharm ${ }^{\circledast}$ erweitert worden. Die 200-mgFilmtabletten sind zugelassen zur Anwendung in Kombination mit Standardpräparaten von Levodopa/Benserazid oder Levodopa/Carbidopa bei Erwachsenen mit Morbus Parkinson, bei denen „End-of-dose“-Fluktuationen auftreten, die mit diesen Standardpräparaten nicht ausreichend stabilisiert sind.

Nach Informationen von neuraxpharm

\section{Gute Note bei „Öko-Test"}

Das Magazin „Öko-Test“ hat zehn Ginkgo-Präparate zur Behandlung bei Gedächtnisstörungen getestet. Nur Tebonin ${ }^{\circledast}$ konzent $240 \mathrm{mg}$ bekam die Note "gut“. Von den übrigen Produkten erhielt eines die Note „befriedigend“, die restlichen „ausreichend“ oder „mangelhaft". In der pharmakologischen Begutachtung kam es darauf an, ob sich ein Arzneimittel in gut durchgeführten Studien für die beanspruchten Anwendungsgebiete als wirksam erwiesen hat. Ausschlaggebend für die positive Bewertung von EGb $761^{\circledR}$ nannte „ÖkoTest“ das Urteil des IQWiG von 2008, das nach umfangreicher Studienauswertung einen Nutzen für das Therapieziel „Aktivitäten des täglichen Lebens" anerkannte.

Nach Informationen von Schwabe 